Hadron Structure '15

International Journal of Modern Physics: Conference Series

Vol. 39 (2015) 1560096 (7 pages)

(C) The Author(s)

DOI: 10.1142/S2010194515600964

\title{
The study of dp-elastic scattering at the energies from 500 to $1000 \mathrm{MeV} /$ nucleon
}

\author{
A. A. Terekhin*, Yu. V. Gurchin, A. Yu. Isupov, A. N. Khrenov, \\ A. K. Kurilkin, P. K. Kurilkin, V. P. Ladygin, N. B. Ladygina, \\ S. M. Piyadin, S. G. Reznikov and I. E. Vnukov \\ Joint Institute for Nuclear Researches \\ Dubna 141980, Russia \\ *aterekhin@jinr.ru
}

Published 26 November 2015

\begin{abstract}
The results of the differential cross section measurements of dp-elastic scattering at energies from 500 to $1000 \mathrm{MeV} /$ nucleon at Nuclotron JINR are reported. The data were obtained for angels range of $75-120^{\circ}$ in the c.m.s. The results are compared with existing world data.
\end{abstract}

Keywords: dp-elastic scattering; differential cross section.

PACS Numbers: $25.10+\mathrm{s}, 21.45+\mathrm{v}, 25.45 \mathrm{De}$

\section{Introduction}

The study of $d p$-elastic scattering at energies up to $1000 \mathrm{MeV} / \mathrm{n}$ at large angles in the c.m.s. is one of the directions of DSS (Deuteron Spin Structure) project research program. ${ }^{1}$ Now the different theoretical models are developed to describe of the three-nucleon scattering. ${ }^{2-5}$ The experimental material for this reaction covers the energy range from tens to thousands $\mathrm{MeV} / \mathrm{n} .{ }^{6-14}$ The theoretical calculations using not only $2 \mathrm{~N}$ forces but also different $3 \mathrm{~N}$ forces ${ }^{15,16}$ give the best agreement with experimental data. However, the discrepancy between the theory and experiment is increasing with the energy increasing indicating the possibility of relativistic effects. The Glauber scattering theory which takes both single and double interactions a classic approach at the energies higher $400 \mathrm{MeV} / \mathrm{n} .{ }^{17,18}$ Relativistic impulse approximation calculations do not describe these data. ${ }^{19}$ The experimental data can be fit with a relativistic multiple-scattering theory which uses off-mass-shell extrapolations of the nucleon-nucleon amplitudes suggested by the structure of derivative meson-nucleon couplings. ${ }^{20}$

This is an Open Access article published by World Scientific Publishing Company. It is distributed under the terms of the Creative Commons Attribution 3.0 (CC-BY) License. Further distribution of this work is permitted, provided the original work is properly cited. 


\section{A. Terekhin et al.}

The experimental setup at internal target station (ITS ${ }^{21}$ at Nuclotron allows to obtain the different observables in the anglular range $60-140^{\circ}$ in the c.m.s. The new preliminary differential cross section data were obtained at ITS Nuclotron at the energies from 250 to $440 \mathrm{MeV} / \mathrm{n}^{22}$ Recently, the deuteron vector and tensor analyzing powers have been obtained at $440 \mathrm{MeV} / \mathrm{n} .{ }^{23}$ The preliminary data of differential cross section for dp-elastic scattering at energies from 500 to $1000 \mathrm{MeV} / \mathrm{n}$, obtained at ITS at Nuclotron are presented in this paper.

\section{Experiment and Data Analysis}

The measurements were performed at $\operatorname{ITS}^{21}$ at the Nuclotron JINR by using $10 \mu m$

$\mathrm{CH}_{2}$ and $8 \mu \mathrm{m} \mathrm{C}$ targets. New ITS DAQ system was used during the data taking. ${ }^{24}$ The elastically-scattered deuterons and protons were registered by two pairs of scintillation detectors placed symmetrically with respect to the beam direction. Also two scintillation counters (PP-detectors) were used to register the quasi-elastically scattered protons. All counters based on the Hamamatsu H7416MOD PMT previously were used in the experiment. ${ }^{23}$ The layout of the counters with respect to the beam direction is shown in Fig. 1.

The $\mathrm{D}_{1,2}, \mathrm{P}_{1,2}$ and $\mathrm{PP}_{1,2}$ are deuteron-, proton- and PP-detectors, respectively. All the counters were placed in horizontal plane. The DP-detectors were rotated to give an angular range of the $\theta_{l a b}=19^{\circ}$ to $50^{\circ}\left(\theta_{\text {c.m. }}=70^{\circ}\right.$ to $\left.120^{\circ}\right)$. The PPdetectors were mounted at the angle corresponding to quasi-elastic scattering at $\theta_{\text {c.m. }}=90^{\circ}$ and remained stationary throughout the experiment. The size of the D-, $\mathrm{P}$ - and PP-counters are $10 \times 40 \times 24 \mathrm{~mm}^{3}, 20 \times 60 \times 20 \mathrm{~mm}^{3}$ and $50 \times 50 \times 20 \mathrm{~mm}^{3}$, respectively. The distances from proton-, deuteron- and PP-counters to the point of the beam interaction with the target are 58,56 and $56 \mathrm{~cm}$, respectively.

In the case of the measurements at $1000 \mathrm{MeV} / \mathrm{n}$ the size of the $\mathrm{D}-, \mathrm{P}$ - and PPcounters are $50 \times 50 \times 20 \mathrm{~mm}^{3}, 20 \times 60 \times 20 \mathrm{~mm}^{3}$ and $\phi 100 \times 200 \mathrm{~mm}^{3}$, respectively. The distance between $P P_{1,2^{-}}$counters and point the beam interaction with the target is $100 \mathrm{~cm}$. Each of such a counter consists of the $\Delta E$ and $E$-detectors. ${ }^{25}$

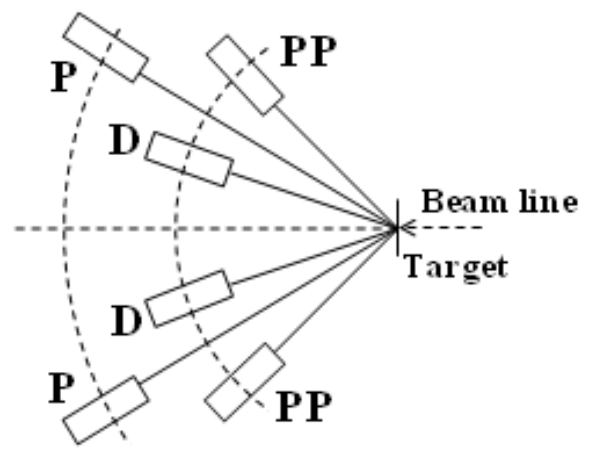

Fig. 1. Layout of the counters with respect to the beam direction. $D_{1,2}, P_{1,2}$ - deuteron and proton detectors, $P P_{1,2}$ - detectors to register of the pp-quasi-elastic scattering. 

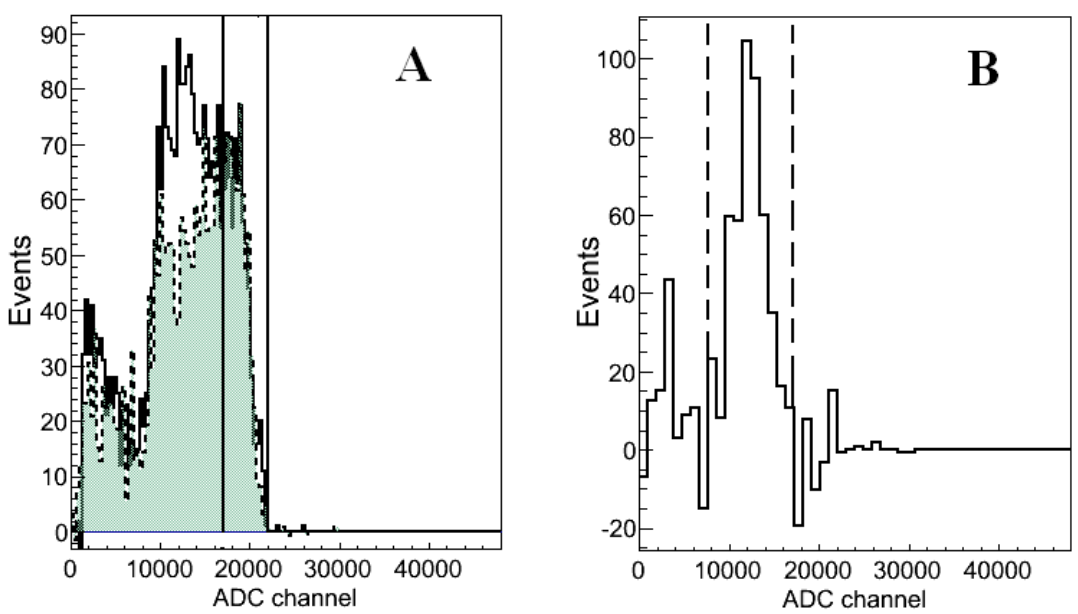

Fig. 2. The procedure of $\mathrm{CH}_{2}-C$ - subtraction for $\theta_{\text {c.m. }}=70^{\circ}$ in the c.m.s. at $1000 \mathrm{MeV} / \mathrm{n}$. A is the $\mathrm{CH}_{2}$ - and normalized $C$-distributions given by the solid and dotted histograms,respectively, vertical solid lines - is the interval of the normalization. B - is the result of $\mathrm{CH}_{2}-\mathrm{C}$ subtraction, vertical dashed lines are the window indicating the domain of the $d p$-elastic scattering events.

The VME based data acquisition system was used for the data taking from scintillation detectors. ${ }^{26}$ TQDC16 module allows to measure the amplitude and time appearance of the signal.

The data analysis was performed in the following way. The selection of the $d p$ elastic scattering events was performed by using the graphical cut for amplitude correlation spectra and the temporary gates on the deuteron and proton time difference spectra. ${ }^{27}$ The next stage is the $\mathrm{CH}_{2}-\mathrm{C}$ subtraction procedure (Fig. 2), the description of which can be found in Ref. 28. The subtraction resulting spectra allows to obtain the dp-elastic scattering events.

The determination of the detectors misalignment was performed at energy $700 \mathrm{MeV} / \mathrm{n}$.

The measurements were performed for each scattering angle in the c.m.s. Since the angle span of the deuteron counter in the c.m.s. is wider than the angle span of the proton counter the deuteron counter is fixed at kinematical angle in the lab. sys. but the proton counter is moved around corresponding angle. The simulation of dp-elastic scattering events by using the Pluto package ${ }^{29}$ was performed. The statistics in this case is reduced about two times if the shift of the detector equals $2^{\circ}$ in the c.m.s. Also the measurements to obtained of the dp-elastic scattering yields were performed (Fig. 3). The founded misalignment equal $1-2^{\circ}$ in lab.sys.

\section{The Differential Cross Section}

In Fig. 4 the data for differential cross section at $1000 \mathrm{MeV} / \mathrm{n}$ are compared with world data and with the theoretical predictions. ${ }^{30}$ New data are shown by the solid 


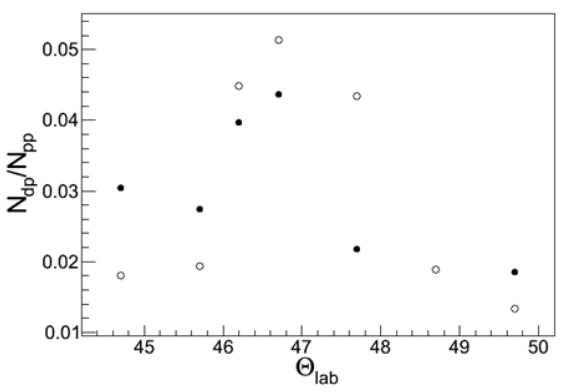

Fig. 3. The experimental relative yields for proton detectors as a function of detector angle for $\theta_{\text {c.m. }}=75^{\circ}$ in the c.m.s. at $700 \mathrm{MeV} / \mathrm{n}$. Filled and open symbols correspond to yields for one and other proton detectors.

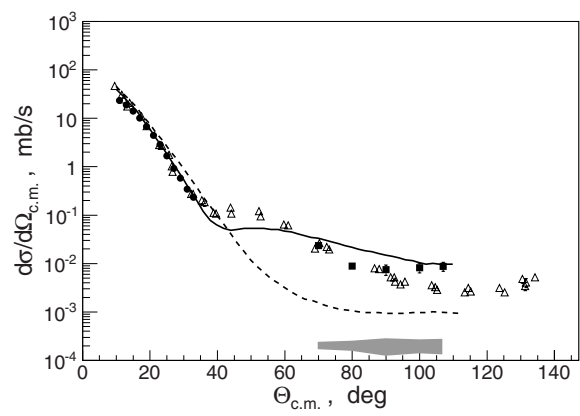

Fig. 4. The differential cross section for $d p$-elastic scattering at $1000 \mathrm{MeV} / \mathrm{n}$. Squares - the results of this work, solid band - the systematic errors, circles - data from Ref. 11, triangles - data from Ref. 13. The dashed and solid lines are the calculations without and with DS term, respectively.

squares. The errors are the statistical only. The systematic error due to normalization and $\mathrm{CH}_{2}-\mathrm{C}$ subtraction procedure $\approx 30 \%$. The data obtained earlier for forward angles ${ }^{11}$ are shown by the solid circles. The open triangles are world data from Ref. 13. The data at Nuclotron are normalized to data from Ref. 13 at $70^{\circ}$ in the c.m.s. One can see that the new data at $\theta_{c . m .} \leq 90^{\circ}$ are in good agreement with the world data. The discrepancy is increasing at large angles, nevertheless, the data are in agreement within the errors which increase with the angle increasing.

Figure 4 shows that the single scattering mechanism does not reproduce the experimental data at the scattering angles $\theta_{c . m}$. larger than $45^{\circ}$. The inclusion of the double scattering (DS) term in the calculations provides better agreement with the experimental results. However, some discrepancy remains. Probably, taking into account new reaction mechanisms like explicit $\Delta$-isobar excitation will improve the description of the data.

The preliminary data for differential cross section at 700,650 and $750 \mathrm{MeV} / \mathrm{n}$ are shown in Figs. 5-7, respectively. One can see a reasonable agreement of the angular behavior of our data with the existing data. ${ }^{10,12}$ 


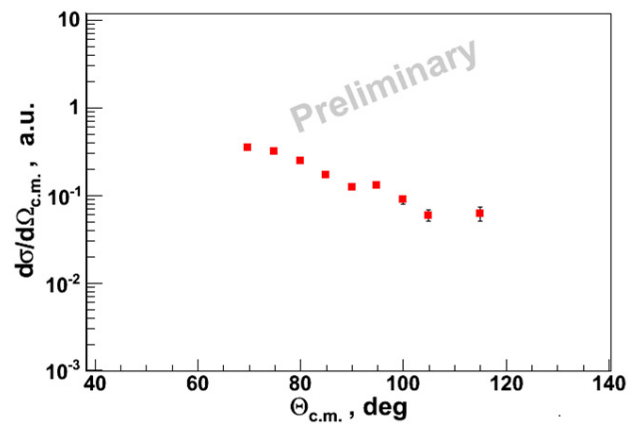

Fig. 5. The angular dependence for differential cross section at $700 \mathrm{MeV} / \mathrm{n}$. The data were obtained by using the results of measurements to determinate of the detectors misalignment for each angle scattering.

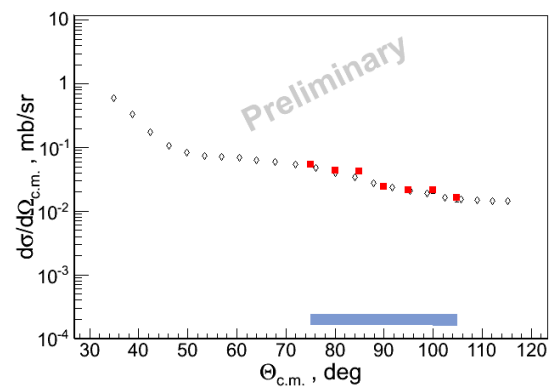

Fig. 6. The differential cross section for dp-elastic scattering at $650 \mathrm{MeV} / \mathrm{n}$. Squares - the results of this work, solid band - the systematic errors, triangles - data from Ref. 10.

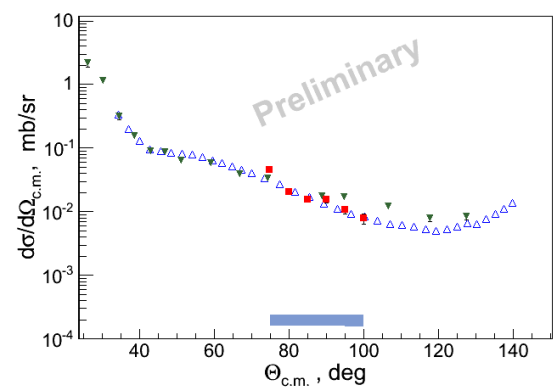

Fig. 7. The differential cross section for $d p$-elastic scattering at $750 \mathrm{MeV} / \mathrm{n}$. Squares - the results of this work, solid band - the systematic errors, open triangles - data from Ref. 10, solid triangles - data from Ref. 12. 


\section{A. Terekhin et al.}

\section{Conclusion}

The procedure on the dp-elastic scattering differential cross section at high energies at ITS at Nuclotron using $\mathrm{CH} 2-\mathrm{C}$ subtraction is established.

The data obtained at $1000 \mathrm{MeV} / \mathrm{n}$ are compared with the calculations performed within the framework of the relativistic multiple scattering theory. It is shown that taking into account the double scattering term improves the description of the obtained experimental results.

The preliminary differential cross section data for dp-elastic scattering at 650 , 700 and $750 \mathrm{MeV} / \mathrm{n}$ are obtained. New results are compared with the existing data for similar values of energies.

The work has been supported in part by the RFBR grant 13-02-00101a.

\section{References}

1. V. P. Ladygin et al., Few-body Studies at Nuclotron-JINR, in Few-Body Systems 55 (2014) pp. 709-712.

2. J. L. Friar et al., Benchmark solutions for n-d breakup amplitudes, Phys. Rev. C51, (1995) p. 2356.

3. A. Kievsky, M. Viviani and S. Rosati, Polarization observables in $\mathrm{p}-\mathrm{d}$ scattering below 30-MeV, Phys. Rev. C64 (2001) 024002.

4. M. Viviani, A. Kievsky and S. Rosati, The Kohn variational principle for elastic proton-deuteron scattering above deuteron breakup threshold, Few-Body Syst. 30, (2001) p. 39.

5. A. Deltuva et al., Benchmark calculation for proton-deuteron elastic scattering observables including Coulomb, Phys. Rev. C 71 (2005) 064003.

6. K. Ermisch et al., Systematic investigation of three-nucleon force effects in elastic scattering of polarized protons from deuterons at intermediate energies, Phys. Rev. C 71 (2005) 064004.

7. K. Sekiguchi et al., Complete set of precise deuteron analyzing powers at intermediate energies: Comparison with modern nuclear force predictions, Phys. Rev. C 65 (2002) 034003.

8. J. C. Alder et al., Elastic pd scattering at $316,364,470$ and $590 \mathrm{MeV}$ in the Backward Hemisphere, Phys. Rev.C 6(6) (1972) 2010.

9. J. S. Vincent et al., Large-angle $p-d$ scattering at $580 \mathrm{MeV}$, Phys. Rev. Lett. 24(5) (1970) 236.

10. E. Culmez et al., Absolute differential cross section measurements for proton-deuteron elastic scattering at 641.3 and $792.7 \mathrm{MeV}$, Phys. Rev. C 43(5) (1991) 2067.

11. A. A. Terekhin et al., Measurement of the differential cross-section and deuteron vector analyzing power in $d p$-elastic scattering at 2.0-GeV, Eur. Phys. J. A 48 (2012) 182.

12. E. Winkelmann et al., Proton-deuteron elastic scattering at $800 \mathrm{MeV}$, Phys. Rev. C 21 (1980) 2535.

13. G. W. Bennet et al., Proton-deuteron scattering at $1 \mathrm{BeV}$, Phys. Rev. Lett. 19 (1967) 387.

14. P. K. Kurilkin et al., New data on the differential cross section of the $d p$-elastic scattering at $2.5 \mathrm{GeV}$ obtained with HADES detector, in the XXI-st International Baldin Seminar on High Energy Physics Problems (ISHEPP 2012) PoS Baldin-ISHEPP-XXI 040 (2012). 
15. S. A. Coon and H. K. Han, Revorking the Tucson-Melbourne Three-Nucleon Potential, Few-Body Systems 30 (2001) 131.

16. B. S. Rudliner et al., Quantum Monte Carlo calculations of nuclei with $A \leq 7$, Phys. Rev. C 56 (1997) 1720.

17. V. Franco and R. J. Glauber, Small-Angle High-Energy Scattering by Deuterons, Phys. Rev. Lett. 16 (1966) 944.

18. V. Franco and E. Coleman, Double Scattering in High-Energy Elastic Collisions with Deuterons, Phys. Rev. Lett. 17 (1966) 827.

19. J. R. Shepard et al., Relativistic Impulse Approximation For P Nucleus Elastic Scattering, Phys. Rev. Lett. 50 (1983) 1443-1446.

20. E. Bleszynski et al., Spin Observables In Proton Deuteron Elastic Scattering, AIP Conf. 150 (1986) 1208.

21. A. I. Malakhov et al., Potentialities of the internal target station at the Nuclotron. Nucl. Instrum. Methods, Phys. Res. A 440 (2000) 320-329.

22. Yu. V. Gurchin et al., The differential cross-section on $d p$-elastic scattering at 400-880 MeV obtained at Nuclotron, Nucl. Phys. Proc. Suppl. 245 (2013) 271.

23. P. K. Kurilkin et al., Measurement of the vector and tensor analyzing powers for $d p$ elastic scattering at $880 \mathrm{MeV}$, Phys. Lett. B 715 (2012) 61-65.

24. A. Yu. Isupov et al., The Nuclotron internal target control and data acquisition system, Nucl. Instrum. Methods Phys. Res. A 698 (2013) 127-134.

25. S. M. Piyadin et al., The study of the $d p \longrightarrow p p n$ reaction at $500-\mathrm{MeV}$ of the deuteron energy at ITS nuclotron, Nucl. Phys. Proc. Suppl. 219-220 (2011) 251.

26. http://afi.jinr.ru

27. A. A. Terekhin et al., The differential cross section in the dp-elastic scattering at the energies from 500 to $1000 \mathrm{MeV} /$ nucleon, in the XXII-st International Baldin Seminar on High Energy Physics Problems (ISHEPP 2015) PoS BaldinISHEPPXXII (2015) 099 (2014).

28. A. A. Terekhin et al., Study of the dp-elastic scattering at $2 \mathrm{GeV}$, arXiv:1503.07968.

29. I. Froehlich et al., A versatile method for simulating $p p \longrightarrow p p e^{+} e^{-}$and $d p \longrightarrow$ pne $e^{+} e^{-} p($ spec $)$ reactions, Eur. Phys. J. A 45 (2010) 401-411.

30. N. B. Ladygina et al., Differential Cross Section of DP-Elastic Scattering at Intermediate Energies, Eur. Phys. J. A 42 (2009) 91. 